\title{
Environmental risk factors and the development of trachomatous trichiasis in Dàlocha District, Central Ethiopia: a case-control study
}

\author{
Ameha Bogale, Abebe Bejiga
}

\begin{abstract}
Background: Trachoma is the second leading cause of blindness in Ethiopia. Even in trachoma hyperendemic areas, not all member of the community develop trichiasis. Whether this is due to uneven exposure or other factors is not clear. This necessitated a case-control study to see the extent of exposure among these groups.

Objective: The purpose of the study was to assess the extent of exposure to known environmental risk factors among cases with trachomatous trichiasis and age, sex and location matched controls with no trachomatous trichiasis in Dalocha District; Central Ethiopia.
\end{abstract}

Methods: all patients with clinically diagnosed trachomatous trichiasis (TT) according to the WHO definition for TT and an equal number of sex and location matched controls with no trachomatous trichasis were subjected to an interviewer administered questionnaire consisting of questions assessing family size, number of children raised, frequency of face washing, water sources and distance from it in minutes, location of kitchen, toilet waste disposal, place for cattle at night and some more considered to be related with repeated trachomatous infection.

Results: One hundred ninety seven cases with TT and an equal number of controls were the study subjects. Regression analysis of individual risk factors showed that irregular face washing practice (odds ratio $(\mathrm{OR}))=2.27 ; 95 \%$ confidence interval (Cl):1.48-3.49) and being illiterate $(\mathrm{OR}=0.34 ; \mathrm{Cl}: 0.18-0.64)$ were significantly associated with the development of TT. Regarding face washing, this significance was also maintained in a logistic regression analysis of the variables where the type of water used for washing and the frequency of cooking were also positively associated with TT.

Conclusion: Improved educational status and regular face washing of the community may decrease the likelihood that people will be at risk of developing TT and its blinding complications. [Ethiop.J.Health Dev. 2002;16(3):287-293]

\section{Introduction}

Trachoma, an ocular infection caused by Chlamydia Trachomatis, is the second cause of blindness worldwide (1). It is estimated that 146 million people are affected by active trachomatous inflammatory diseases, and roughly 6 million people are irreversibly blinded or have severe visual impairment from trachoma (2). Although at one time global in

Department of Ophthalmology, Faculty of Medicine, Addis Ababa University, P.O. Box 9086, Addis Ababa, Ethiopia it's distribution, trachoma is now mostly confined to developing countries where community and family living conditions continue to foster it's transmission (3-4).

In Ethiopia, the significance of trachoma as a cause of blindness varies from one community to the other. Accordingly, previous studies conducted in different parts of Ethiopia reported that trachoma contributed to $35 \%$ to $42 \%$ of all blindness in the country $(5,6)$. Overall, it is the second leading cause of blindness responsible for $30 \%$ of blindness in our country (7). A previous study carried out in 
Dalocha District revealed that $3.2 \%$ of the general population and $5.5 \%$ of people older than 10 years had trichiasis (8).

Trachoma is a communicable disease of low infectivity; it requires high frequency and duration of exposure to persist in a population (9). Repeated infection results in scarification and shortening of the inner lamellae of lids causing trichiasis, which in turn causes corneal opacity and blindness. Therefore, the potentially blinding complications of trachoma seen in adults are the consequences of repeated eye infections during childhood (10).

Several risk factors that predispose to trachoma infection have been identified. The major one are female sex, lack of water, cattle ownership, crowding, hygienic practices, low socioeconomic status and sleeping in a room with a cooking fire (11-13). This study was designed to assess the differences in past exposure to presumed risk factors like family size, number of children, face washing habit, type and distance of water source, place for cooking and cattle keeping, use of waste disposal and latrine contributing to repeated attacks of trachoma between cases with trichiasis and controls without trichiasis.

\section{Methods}

The study was carried out in the community of Dalocha District between January and February. 2000. The area is located in Central Ethiopia about 182 kilometers from the capital, Addis Ababa. It is at the transition between a highland and rift valley having an altitude of 1500 to 2000 meters above sea level. There is one health center in Dalocha town. Two eye centers staffed by ophthalmologists exist between 50 to $100 \mathrm{kms}$ form the area i.e., Butajira and Hosanna Hospitals.

The following standard sample size formula for a-cases- control study design was used. Relative risk was taken to be 2 and a prevalence of trichiasis (TT) of $5.5 \%$ obtained from a previous study was used $\mathrm{n}(\mathrm{each}$ group $=(p 0 q 0+p 1 q 1) \quad(z l-a / 2+z 1 B) 2 /\left({ }_{1}-P_{0}\right)^{2}$. Ethical clearance was obtained from the research and publication committee of the Department of Ophthalmology, Faculty of Medicine of Addis Ababa University. The calculated sample size of 197 people with diagnosed trachomatous trichiasis and an equal number of sex, age and location matched controls were included.

All people above the age of 30 years in the study area with self-diagnosed trichiasis were informed by community health agents (CHA) to come to Dalocha Health Center. Diagnosis of trachomatous trichiasis was confirmed and its grading as minor ( $<5$ lashes) and major $(>5$ lashes) was made by an ophthalmic resident and two trained health assistants using torch light and a magnifying loupe $(2.5 x)$. It was noted that all self-diagnosed cases of TT were confirmed to be positive by the ophthalmic resident and health assistants.

Data was collected using an intervieweradministered questionnaire by two trained high school graduate enumerators who were able to speak the local language and had previous experience in similar study in the same area. The enumerators were trained in data collection through questionnaire and also in TT identification. The questionnaire was pretested in the community and the result was discussed to resolve any existing ambiguities. The diagnosis agreement level determined between the enumerators and the senior ophthalmic resident in $\mathrm{TT}$ identification was found to be $92 \%$. Verbal consent was obtained before the administration of the questionnaire.

Some of the factors covered were family size, number of children raised, literacy status, face washing habit, distance from and type of water source, location of kitchen, frequency of cooking, availability of waste disposal and toilet, and place where cattle spend during the night. Face washing was categorized as regular 
or irregular. Regular face washing was defined as atleast once daily practice while irregular face washing was considered if the individual practiced it only when water was available. Cooking practices which were likely to expose individuals to prolonged irritation of smoke were considered. Thus, cooking indicated the preparation of local bread rather than the preparation of coffee or sauces which was assumed to have less exposure time.

At the completion ' of each questionnaire, the enumerators asked the cases for a home address of a neighbour with similar age and sex but having no problem of trichiasis. Then, the enumerators went into the villages to trace and examine the eyes of controls for the presence or absence of trichiasis at their home. If the traced control happens to have TT or is absent, enumerators were allowed to pick and examine any one in the vicinify with similar age and sex to the reference case and administer the questionnaire. All patients found to have TT were surgically corrected at the health center.

Data was entered and analyzed by the EPI info. version 6 statistical package. Proportions, logistic regression and odds ratio were used for the analysis.

\section{Resuits}

A totals of 197 cases with trichiasis and an equal age, sex and location matched controls were studied." As seen in table 1, females constituted $76.6 \%$ making female to male ratio of about $3: 1$. Since this study was conducted in a rural setup, most of the cases and controls were farmers (89\% vs 92\%) as well as illiterate (91\% vs $78 \%$ ). About $95 \%$ of the study population were of Muslim faith. The mean age among cases and controls was 43.8 and 38.1 years respectively. The mean current family size of the two groups was 5.4 and 6.0 for the cases and controls respectively.

Table 1: Comparison of environmental risk factors among cases and controls, Dalocha District, 2000.

\begin{tabular}{lccc}
\hline Variables & Cases $(\mathrm{n}=197)$ & Controls $(\mathrm{n}=197)$ & P-value \\
\hline Female sex & $151(76.6 \%)$ & $151(76.6 \%)$ & 0.14 \\
Farmer & $175(88.8 \%)$ & $182(92.4 \%)$ & 0.30 \\
Muslim religion & $187(94.9 \%)$ & $186(94.4 \%)$ & 1.00 \\
Illiterate & $180(91.4 \%)$ & $154(78.2 \%)$ & 0.00 \\
Irregular face washing & $124(62.9 \%)$ & $85(43.1 \%)$ & 0.00 \\
Kitchen in living room & $185(93.9 \%)$ & $185(93.9 \%)$ & 0.83 \\
No latrine & $189(95.9 \%)$ & $180(91.4 \%)$ & 0.09 \\
No Waste disposal & $183(92.9 \%)$ & $187(94.9 \%)$ & 0.52 \\
Cattle keeping in living room at night & $183(92.9 \%)$ & $183(92.9 \%)$ & 0.43 \\
Daily cooking & $149(75.6 \%)$ & $141(71.6 \%)$ & 0.42 \\
Distance to water <1 hour & $190(96.4 \%)$ & $190(96.4 \%)$ & 0.78 \\
\hline
\end{tabular}

Comparison of the risk factors among cases and controls revealed that the differences in face washing habit $(\mathrm{p}=0.00)$ and literacy status $(p=0.00)$ were found to be statistically significant between the two groups. Occupationally, though $90.6 \%$ of the study population were farmers, its association with
TT was not significant $(\mathrm{OR}=0.66 ; \mathrm{Cl}: 0.31$ 1.38). Availability of latrine was associated with lower risk of TT (4.1\% versus $8.6 \%$ ) but this difference was not statistically significant because of the low rates of latrine in the population. 
Logistic regression (table 2) analysis of the variables showed that as face washing practice improves, the prevalence of TT correspondingly declines. The odds of being literate and developing TT was also significantly low as compared to controls
(OR $=0.34 ; 95 \%$ CI:0.18-0.64). Being female $(\mathrm{OR}=1.44)$, daily cooking $(\mathrm{OR}=1.23)$, cattle ownership (OR=1.21), use of pond water for cleaning $(\mathrm{OR}=1.20)$ and age more than 30 years $(O R=1.13)$ all seemed to carry higher risk for TT, but these differences were not significant.

Table 2: Results from logistic regression analysis of risk factors for trichiasis in Dalocha District, 2000

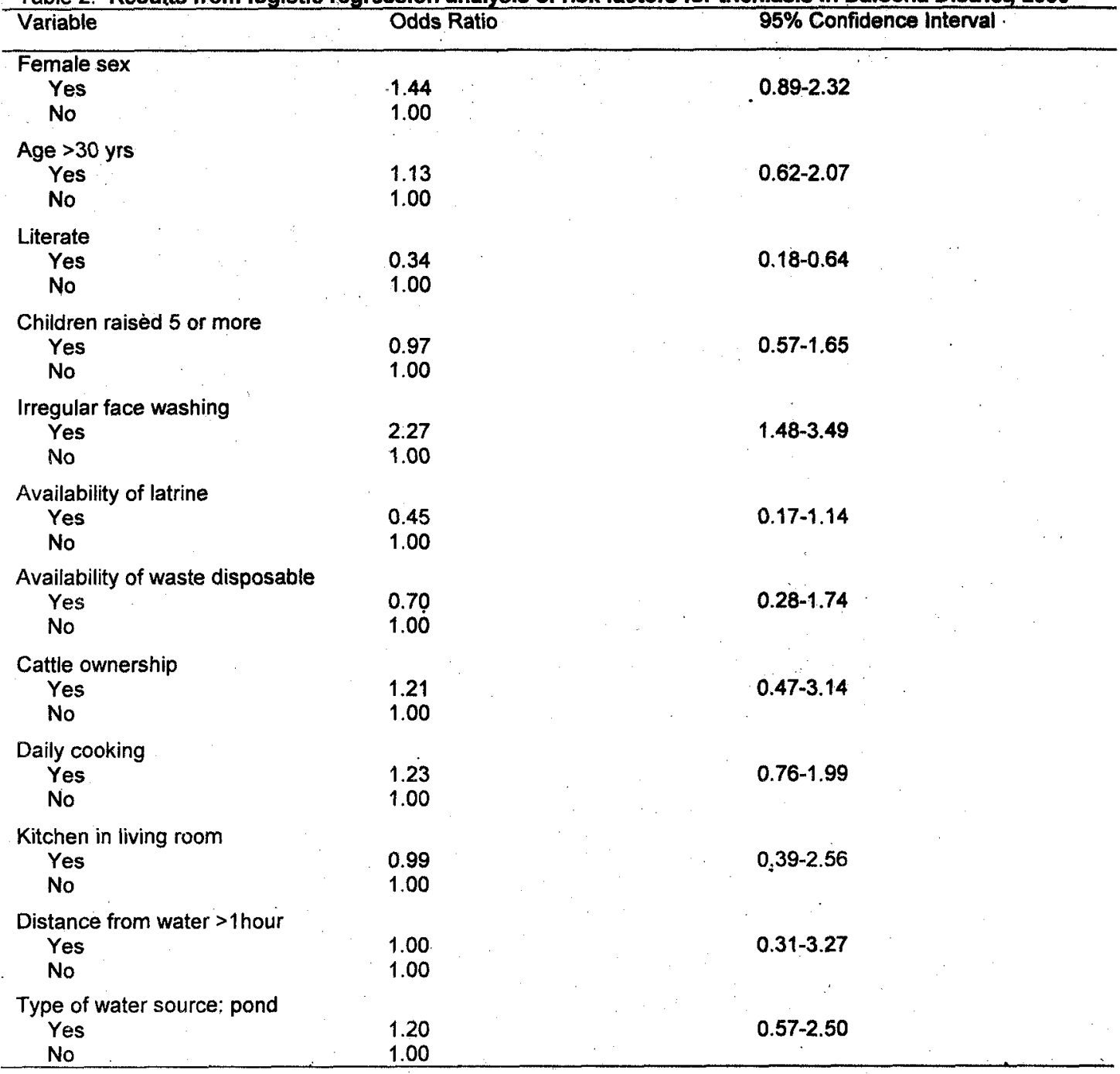


Though the average number of children raised was 6.4 for the cases and 6.2 for the controls, this was not significantly different $(O R=0.97$; CI:0.57-1.65). There was no difference between the cases and controls regarding distance from water source $(\mathrm{OR}=1.00 ; 95 \%$
CI:0.31-3.27). The type of water source used for washing seemed more important than distance from it as seen in table 3 . The reason may be that water in ponds is seasonal depending on rain to sustain its availability throughout the year.

Table 3: Type of water source and its association with trichiasis among the study population, Dalocha District, 2000

\begin{tabular}{llccc}
\hline Source & Cases & Controls & Odds Ratio & $95 \%$ Cl \\
\hline Pipe & $148(75.1 \%)$ & $148(75.1 \%)$ & 1.00 & $0.61-1.63$ \\
Pond & $20(10.2 \%)$ & $17(8.6 \%)$ & 1.20 & $0.57-2.50$ \\
River & $24(12.2 \%)$ & $32(16.2 \%)$ & 0.72 & $0.39-1.32$ \\
Well & $5(2.5 \%)$ & $0(0 \%)$ & & \\
\hline Total & $197(100 \%)$ & $197(100 \%)$ & & \\
\hline
\end{tabular}

\section{Discussion}

The predominantly female population (76.6\%) in this study; though not randomly selected to be representative, may be considered as reflecting the picture of the community. It has been reported (14) that about $75 \%$ of the cases of trichiasis were females. This has been explained to be due to their close association with and responsibility of caring for children who are reserviour for the infection. In addition to child care, cooking which by way of its irritant effect is said to aggravate the severity of trachomatous inflammatory process leading consequently to trichiasis, is the domain of females in our culture. However, eventhough female sex was associated with a higher risk of developing trichiasis, the difference was not significant in this study ( $\mathrm{OR}=1.44 ; 95 \%$ CI:0.89-2.32).

In this study, the type of water used for washing face seemed more important than the distance from its source. The use of pond water, though not significantly different, had higher risk for TT (OR=1.20;CI:0.57-2.50) compared to pipe and river sources. Similar finding was reported from Nepál previously (12). This may be due 'to' the fact that water availability in ponds is variable with the time of the year leading to its. shortage during dry seasons. Above all, poor face washing habit and being illiterate were found to be the most significantly associated factors with the development of trichiasis in this study. This goes with a Tanzanian report that revealed clean face (free from flies and nasal discharge) to be associated with lower prevalence of trachoma (15). As this issue has been a well recognized preventive method included in the SAFE strategy, our study also emphasizes the need for health education as related to individual hygiene in the prevention of trachoma and its complications.

A study carried out in the southern and eastern lowlands of Somali, Kereyou and Borena to examine the impact of cattle raising on the prevalence and severity of trachoma has indicated that cattle droppings may play an important role in the transmission of eye infection, particularly trachoma, by furnishing rich breeding site for flies (16). Regarding cattle; their droppings attract flies which are transmitters of trachoma infection. Furthermore, it was a common practice to keep cattle with people in the living room during the night to keep them secured from thieves and offending wild animals in the study area. Though cattle ownership has been reported to be associated with higher risk of trachoma (911), this was not found to be significantly different between cases and controls in our study (OR=1.21;CI:0.47-3.14). 
Other risk factors for trichiasis considered in this study were family size, number of children cared for, location of kitchen, ownership of latrine and waste disposal. Trachoma is said to cluster within families and transmission of infection commonly occurs within family members (17). This implies that once infection has occurred in a particular family, the risk of repeated infection for the mother or care-taker who are mostly females increase with the number of child raised. Increased family size and overcrowding as being associated with the occurrence of trachoma infection is also explained on the basis that infection runs among family members. Though the number of children raised is one risk factor which predisposes mothers to the development of TT in trachoma endemic areas, this was shown to be similar among cases and controls.

This result by no means imply that living under the known predisposing environmental condition doesn't lead to trachoma and its complications but at least indicate that at the age when susceptible groups are supposed to develop chronic complications of trachoma some individuals didn't. The study has its own limitations on the strength of its measurements used to assess many of the assumed risk factors. Factors like water source, access or distance from water and face washing habit need more detailed measurement techniques better than the ones we used in our questionnaires. Other factors such as the socioeconomic status and per capita water consumption which we didn't look for will require further investigation. We, therefore, suggest a further study about the strength of exposure these unaffected individuals really had to these risk factors.

\section{Acknowledgements}

We would like to thank ORBIS InternationalEthiopia Country Office for funding the research. Our special thanks goes to Dr. Muluken Melese, MPH and Dr. Wondu Alemayehu MPH for their technical advice and continuous supervision. We appreciate the support given by the staff of Action Aid Ethiopia Dalocha Development Areas, Dalocha Health Center and Dalocha Administration.

\section{References}

1. West SK, Munoz B, Lynch M, Kayongoya A, Mmbaga $\mathrm{BBO}$ and Taylor HR. Risk factors for constant, severe trachoma among preschool children in Kongwa, Tanzania. Am J Epidemiol 1996;143:73-8.

2. Thylefors B. Trachoma new opportunities to tackle an old problem. Br J Ophthalmol Editorial.

3. Dawson $\mathrm{Cr}$ et al guide to trachoma control. Geneva, WHO, 1981.

4. Thylefors B. Development of trachoma control program and the involvement of natural Resources. Reviews of Infectious Diseases. 1985;7:774-776.

5. Wondu A. Redda TH, Fosegren L and Ekstedt J. Causes of visual Impairment in Central Ethiopia. Ethiop Med J 1995;33(3): 163-174.

6. Budden F. Blindness in Ethiopia. Report to WHO, Geneva; 1981 .

7. Guideline for the prevention of blindness programmes in Ethiopia, Ministry of Health, 1996.

8. Abebe $B$ and Wondu A. Prevalence of trachoma and its determinants in Dalocha District, Central Ethiopia. Ophthalmic Epidemiology 2001;8(2-3):119-125.

9. Schwab L et al. The epidemiology of trachoma in rural Kenya: variation in prevalence with lifestyle and environment. Ophthalmology 1995; 102:475-82.

10. Munoz B. Incidence estimates of late stages of trachoma among women in hyperendemic area of central Tanzania. Tropical Medicine and International Health 1997;2(11):1030-8.

11. Turner VM et al. Risk factors for trichiasis in women in Kongwa, Tanzania: A casecontrol study. International Journal of Epidemiology 1993;22(2):341-7. 
12. Katz J et al. Prevalence and risk factors for trachoma in Sarlahi district, Nepal. AM J Ophthalmol 1996;80:1037-1041.

13. Sole G. Impact of Cattle on the Prevalence and. Severity of Trachoma. $\mathrm{Br}$ I Ophthalmol 1987;71:

14. Taylor HR. The natural history of endemic trachoma: a longitudinal study. Am J Trop Med Hyg 1992;45(5):552-9.

15. West SK et al. Impact of face washing on trachoma in Kongwa, Tanzania. Lancet 1995;345:155-8.
16. Sahelu $\mathrm{T}$ and $\mathrm{C}$ Larson. The prevalence and environmental risk factors for moderate to severe trachoma in southern Ethiopia. J Tropical Medicine and Hygiene. 1992;95:36-41.

17. West SK et at. The epidemiology of trachoma . in central Tanzania. International Journal of Epidemiology 1991;20(4):1088-92. 